\title{
Polymyxin-B Immobilized Fiber PMX-20R
}

National Cancer Institute

\section{Source}

National Cancer Institute. Polymyxin-B Immobilized Fiber PMX-20R. NCI Thesaurus. Code C135615.

A cartridge containing a polystyrene-based composite woven fiber that is covalently attached to the anti-microbial cyclic cationic polypeptide antibiotic polymyxin-B (PMX) and can potentially be used for hemoperfusion purposes. Upon extracorporeal treatment, a patient's blood flows through the fibers in the polymyxin-B immobilized fiber PMX-20R cartridge and the circulating endotoxins from the blood are selectively adsorbed by PMX, which has positively charged amino groups that bind to the negatively charged site in the lipid A portion of the lipopolysaccharide (LPS) layer of the bacterial cell membrane. This removes endotoxins from the blood and, upon administration of the blood back into the patient, helps treat or reduce the incidence of endotoxemia and sepsis. 